\title{
A NOTE ON HARMONIC FUNCTIONS AND A HYDRODYNAMICAL APPLICATION
}

\section{HANS LEWY}

In this note I show how the method by which I recently proved ${ }^{1}$ the analytic extensibility of a minimal surface across an analytic boundary portion can also be used to prove the following (considerably simpler) theorem.

TheOREM. Let $U(x, y)$ be harmonic near the origin in $y<0$, and $V(x, y)$ a conjugate harmonic of $U$. Assume that $U, V, \partial U / \partial x$ exist and are continuous in the semi-neighborhood $y \leqq 0$ of the origin. If the boundary values on $y=0$ satisfy a relation

$$
\partial U / \partial y=A(x, U, V, \partial U / \partial x)
$$

in which $A$ is an analytic function of all four arguments for all values occurring, then $U(x, y)$ and $V(x, y)$ are analytically extensible across $y=0$.

Proof. Set $z=x+i y, 2 F(z) \equiv 2 F(x, y) \equiv U(x, y)+i V(x, y)$. Introduce a function $G(x, y) \equiv G(z)$ through the following construction: $2 G(0,0)=U(0,0)-V(0,0) i$ and $G$ satisfies on $x=0, y \leqq 0$ the differential equation

$$
\begin{aligned}
\partial G(0, y) / \partial y & =\partial F / \partial y-A[y i, G(0, y)+F(0, y), \\
& i(G(0, y)-F(0, y)),-i(\partial / \partial y)(G(0, y)+F(0, y))],
\end{aligned}
$$

and on lines $y=$ const. $\leqq 0$ the equation

$$
\begin{aligned}
\partial G(x, y) / \partial x=\partial F / \partial x+i A[x+y i, F(z)+G(z), \\
i(G(z)-F(z)),(\partial / \partial x)(F(z)+G(z))] .
\end{aligned}
$$

It is immediately seen that the construction with the aid of $\left(\mathrm{E}_{y}\right)$ falls within the scope of the classical existence and uniqueness theorem for ordinary differential equations provided $\left(\mathrm{E}_{y}\right)$ can be solved with respect to $\partial G / \partial y$ which occurs on both sides of the equation. To see this, observe that it suffices that at the initial point $(0,0)$ the partial derivative of the left-hand side of $\left(E_{y}\right)$ with respect to $\partial G / \partial y-$ of value $1-$ differ from that of the right-hand side which, at the origin, equals $i \partial A / \partial U_{x}$ and is a pure imaginary there, if we

Presented to the Society, April 28, 1951; received by the editors March 15, 1951.

${ }^{1}$ On the boundary behavior of minimal surfaces, Proc. Nat. Acad. Sci. U.S.A. vol. 37 (1951) pp. 103-110. 
take $\partial G / \partial y=-\partial F / \partial y+i \partial U / \partial x$ at the origin as is consistent with (B) and $\left(E_{y}\right)$.

Having thus established $G(0, y)$ for $y \leqq 0$ near the origin as solution of $\left(\mathrm{E}_{y}\right)$, we utilize $\left(\mathrm{E}_{x}\right)$ similarly for extending $G$ on the $x$-axis and parallels to it by choosing as initial values the found values of $G(0, y)$. Again the solution exists and is uniquely determined if we make sure that $\partial G(0, y) / \partial x=-i \partial G(0, y) / \partial y$, which is always consonant with $\left(\mathrm{E}_{x}\right)$ and $\left(\mathrm{E}_{y}\right)$.

Now take a value $y_{0}<0$ such that the point $i y_{0}$ is contained within the domain of the above construction. Consider the solution $G(z)$ of the equation

$$
d G / d z=d F / d z+i A(z, F+G, i(G-F), d G / d z+d F / d z),
$$

for which $G\left(i y_{0}\right)=G\left(0, y_{0}\right),(d G / d z)_{z-i y_{0}}=-i G_{y}\left(0, y_{0}\right)=G_{x}\left(0, y_{0}\right)$, in accordance with $(E)$. The solution of this initial problem for $(E)$ is uniquely determined and is a regular complex function of $z$ at all interior points of its domain of existence. On the other hand, on the $y$-axis $(\mathrm{E})$ becomes $\left(\mathrm{E}_{y}\right)$ by specialization of the derivative $d G / d z$, and on parallels to the $x$-axis $(E)$ similarly becomes $\left(E_{x}\right)$; it follows that the uniquely determined solution of $(E)$ is no other than our above $G(x, y)$.

The $x$-axis is part of the boundary of the domain of construction, hence the analyticity of $G(z)$ does not a priori follow for points of the $x$-axis. However, we know that by hypothesis $F$ and $\partial F / \partial x$ remain continuous up to the $x$-axis. Thus, the equations $\left(\mathrm{E}_{x}\right)$ may be considered as ordinary differential equations for $G(x, y)$, depending continuously on the parameter $y$ as $y \rightarrow 0$, and the initial values $G(0, y)$ depend likewise continuously on the parameter $y$. Thus the solutions $G(x, y)$ tend uniformly to $G(x, 0)$ as $y \rightarrow 0$, again by an application of the classical continuity theorem for ordinary differential equations. Now $G(x, 0)$ is a solution of $\left(\mathrm{E}_{x}\right)$ with initial value $G(0,0)=\bar{F}(0)$, where the bar indicates the complex conjugate; but a solution with the same initial value is certainly $G(x, 0)=\bar{F}(x)$ since this substitution changes $\left(\mathrm{E}_{x}\right)$ into (B). Thus uniqueness requires $G(x, 0)=\bar{F}(x)$.

Now consider, for $y \geqq 0$, the definition

$$
F(x+i y)=\bar{G}(x-i y) .
$$

Evidently this $F$ is regular in $z$ for $y>0$ since $G(x-i y)$ is a regular function of $x-i y$. The new definition agrees with the one previously given on the $x$-axis. Thus, by a well known theorem, the new definition is the analytic extension of $F(z)$ across the $x$-axis. Our theorem is proved. 
To illustrate the usefulness of the theorem we shall apply it to the irrotational steady flow of a homogeneous incompressible liquid in the neighborhood of a free boundary where the pressure is constant and Bernoulli's law holds:

$$
T+V=\text { const.; }
$$

here $T$ is the kinetic energy per unit mass, $V$ the potential energy of gravity per unit mass. This example includes the case of progressive water waves in which the profile of the surface advances without change of form and with uniform velocity, provided the motion is referred to a Galilei co-ordinate system with respect to which it becomes steady.

Since $T \geqq 0$, there derives from Bernoulli's law a bound for the elevation of the free surface, which bound is obtained by setting formally $T=0$. If we point the $y$-axis in the opposite direction of gravity, and put its origin at the just mentioned elevation, and if we further adjust the units of mass and length appropriately, the Bernoulli law takes at the free surface the simple form:

$$
T=-y .
$$

Denote by $\phi, \psi$ velocity potential and stream function, set $\zeta=\phi$ $-i \psi, z=x+i y$, and adjust the additive constant in $\psi$ so that the free surface becomes the line $\psi=0$. As long as $T>0$, we have $y<0$ and it is legitimate to replace (locally) the independent variable $z$ by $\zeta$. For the Bernoulli relation becomes on $\psi=0$

$$
-2 y|d z / d \zeta|^{2}=1 \text { or }-2 y\left[(\partial y / \partial \phi)^{2}+(\partial y / \partial \psi)^{2}\right]=1 .
$$

As $y$ is a harmonic function of $\phi, \psi$, our theorem is applicable and we conclude that $y(\phi, \psi)$ is an analytic function of $\phi$ for $\psi=0$. Similarly the conjugate harmonic function $x(\phi, \psi)$ is analytic for $\psi=0$, by the same theorem. The assumption has been made that the velocities of the particles are continuous functions of the point on and near the free surface.

If the assumption $T>0$ is dropped, the possibility that $y=0$ arises. If this happens at an isolated point $P$, then the Bernoulli relation $\left(\mathrm{B}^{\prime}\right)$ is no longer solvable at $P$ with respect to $\partial y / \partial \psi$ or $\partial x / \partial \psi=-\partial y / \partial \phi$ in analytic manner and our theorem no longer applies. As Stokes has pointed out, the corresponding wave may have at $P$ a cusp of angle $2 \pi / 3$.

STANFORD UNIVERSITY 